\title{
Satisfação de Profissionais de Educação Física na Regência em Aulas de Ginástica de Academia
}

\author{
Satisfaction of Physical Education Professionals at Teaching Fitness Classes
}

\section{Satisfacción de Profesionales de Educación Física en la Regencia en Clases de Gimnasia de Academia}

\author{
Ana Luiza Barbosa Anversa ${ }^{1}$ \\ Tieli Fernanda Ribeiro \\ Luciana Boligon Refundini \\ Daniel Vicentini de Oliveira
}

Centro Universitário Metropolitano de Maringá (UNIFAMMA)

\begin{abstract}
Resumo
Este estudo teve como objetivo analisar a satisfação de profissionais de Educação Física na regência em aulas de ginástica de academia. Pesquisa transversal, realizada com 20 professores de ginástica de academia, em que foi utilizado o "Instrumento para a medida de satisfação no trabalho". A análise dos dados foi realizada por meio dos testes de Shapiro-Wilk e $t$ de Student independente. Considerou-se o nível de significância de $p<0,05$. O nível total de satisfação no trabalho foi considerado regular entre os professores. Ao comparar os níveis em relação ao gênero, encontrou-se diferença significativa nos âmbitos fisiológicos $(p=0,034)$ e segurança $(p=0,043)$, evidenciando que as mulheres apresentaram melhores escores quando comparadas com os homens. A maioria dos profissionais de Educação Física atuante na ginástica de academia mostrou-se satisfeita em ministrar essas aulas, destacando-se atenção para os âmbitos fisiológicos e de segurança, entre os homens.
\end{abstract}

Palavras-chave: atividade motora, ginástica, satisfação

\begin{abstract}
This study aimed to analyze the satisfaction of physical education professionals at teaching fitness classes. Cross-sectional research, carried out with 20 fitness center instructors. An "Instrument to measure satisfaction at work" was used. Data analysis was performed using the Shapiro-Wilk test and independent Student t-test. The level of significance adopted was $p<0.05$. The total level of satisfaction was considered regular among the instructors. When comparing levels in relation to sex, a significant difference was observed in the domains "physiological aspects" ( $p=0.034)$ and "safety" ( $p=0.043)$, showing that women had better scores when compared to men. The majority of physical education professionals working as instructors in fitness centers were satisfied to give these classes, emphasizing the scopes of "physiological aspects" and "safety" among men.

Keywords: motor activity, fitness, satisfaction

\section{Resumen}

Este estudio tuvo como objetivo analizar la satisfacción de profesionales de educación física en la regencia en clases de gimnasia de academia. Investigación transversal, realizada con 20 profesores de gimnasia de academia. Se utilizó el "Instrumento para la medida de satisfacción en el trabajo". El análisis de los datos fue realizado por medio de la prueba de Shapiro-Wilk y t de student independiente. Se consideró el nivel de significancia de $p<0,05$. El nivel total de satisfacción en el trabajo fue considerado regular entre los profesores. Al comparar los niveles en relación al sexo, se encontró una diferencia significativa en los ambitos fisiológicos $(p=0,034)$ y de seguridad $(p=0,043)$ evidenciando que las mujeres presentaron mejores puntuaciones cuando se compararon con los hombres. La mayoría de los profesionales de Educación Física actuante en la gimnasia de academia se mostró satisfecha en ministrar esas clases destacándose la atención a los ámbitos fisiológicos y de seguridad, entre los hombres.

Palabras clave: actividad motora, gimnasia, satisfacción
\end{abstract}

\footnotetext{
${ }^{1}$ Endereço de contato: Avenida Mauá, 2854, zona 03. 87013-160 Maringá, PR. E-mail: ana.beah@gmail.com
} 


\section{Introdução}

Os profissionais de ginástica de academia apresentam uma rotina emocional e fisicamente estressante, caracterizando uma exaustão profissional, causada por longas horas de trabalho, busca da satisfação das necessidades dos clientes e excessos de trabalho. Tal esgotamento acarreta a falta de satisfação (Brooks, 2007) ou ainda está relacionado com valores, atitudes, percepção de justiça de remuneração e jornada de trabalho (Siqueira \& Gomide Júnior, 2004).

As academias de ginástica, em sua maioria, são espaços com fins lucrativos, indicados para quem busca a prática de atividade física, sob orientação de profissionais de Educação Física, formados e capacitados para atender os alunos (clientes) em seus anseios e necessidades (Saba, 2001). As aulas de ginástica em grupo ofertadas nestes espaços têm como objetivo trabalhar o condicionamento físico, aliando música e movimento, para propiciar o bem-estar e fins estéticos. Para o profissional, é um desafio constante promover necessidades impostas por novos padrões de beleza, atendendo aos anseios dos alunos (Moreira, Freitas, Guarido, \& Bruniera, 2017).

Garay, Silva e Beresford (2011) destacam que o cliente deve ser atendido com uma visão mais ampla, que vai além do movimento, dos exercícios e da repetição, numa dimensão psíquica, social e emocional. No entanto, aqueles profissionais que atuam nas aulas de ginástica em grupos devem estar atentos quanto à motivação dos alunos. É preciso sair da zona de conforto, oferecendo opções para alunos iniciantes e avançados, respeitando os limites de cada um. Para um crescimento do público nessas aulas, devem se evitar situações monótonas e movimentos repetitivos; por isso, o profissional tem de ser prudente quanto ao grau de motivação de seu grupo, uma vez que, quanto mais motivados, melhor será a performance no exercício realizado (Sena, 2015).

As características do profissional de Educação Física regentes nessa área de atuação da ginástica de grupo implicam diretamente em um perfil criativo, extrovertido, comunicativo e competente, nas explicações dos exercícios. Ao dominar sua área da atuação, o profissional fica responsável por fidelizar os alunos, principalmente nas aulas de ginástica.

Assim, para Coelho Filho e Votre (2010), o professor de ginástica em academia deve avaliar o conjunto de princípios e estratégias de organização do ambiente, e os objetivos que devem presidir o ensino e as estratégias adequadas para se alcançarem determinados objetivos adequados aos alunos, pois ministrar uma aula de ginástica de academia não é só executar de forma correta os exercícios para os alunos repetirem, mas também proporcionar uma atividade física segura, eficiente e divertida, orientando e transmitindo conhecimento pela promoção da saúde (Rufino, Soares \& Santos, 2000). O profissional que busca seguir essa linha de atuação tem de trabalhar com equilíbrio, entre seus conhecimentos, visando agradar um grupo heterogêneo. Para tanto, esse profissional necessita de uma motivação e satisfação pessoal, para que haja um elo com os alunos e, assim, se concretizar o bom trabalho. As motivações surgem de nossas necessidades, sejam estas físicas, espirituais, artísticas ou sociais, e têm a ver com os movimentos intencionais e funcionais da personalidade (Feijó, 1998).

Folle, Borges, Coqueiro, e Nascimento (2008) destacam que "a satisfação está relacionada com o sentir-se bem no ambiente de trabalho, e se reflete diretamente no desempenho das suas funções (realização pessoal e profissional)". 
A satisfação profissional é um aspecto que pode influenciar no nível de percepção de bem-estar geral do individuo, por possuir grande influência na determinação dos níveis de estresse (Marqueze, \& Moreno, 2005).

Diante do exposto e considerando-se que o nível de satisfação no trabalho do profissional de Educação Física se reflete diretamente no quadro de aderência e permanência nas aulas de ginástica de academia, e na qualidade da intervenção ofertada, a presente pesquisa teve como objetivo analisar a satisfação de profissionais de Educação Física na regência em aulas de ginástica de academia.

\section{Método}

A presente pesquisa caracteriza-se como quantitativa, transversal e observacional, e foi aprovada pelo Comitê de Ética em Pesquisa, sob o parecer consubstanciado 1.785.188.

\section{Participantes}

A coleta de dados ocorreu em quatro academias do município de Maringá, Estado do Paraná, PR, selecionadas de forma intencional. Nas quatro academias selecionadas, havia 60 profissionais de Educação Física regularmente contratados e cadastrados no Conselho Regional de Educação Física (CREF/PR). Para compor a amostra, foram convidados 20 professores, sendo 11 do gênero feminino e nove do gênero masculino. Como critério de inclusão, os professores deveriam trabalhar na regência de aulas de ginástica em academia havia pelo menos dois anos.

\section{Instrumentos}

Para a coleta de dados, foi utilizado o "Instrumento para a medida de satisfação no trabalho", construído e validado por Hesketh e Costa (1980). O questionário contém 42 questões, subdividias em cinco âmbitos; 1 ) necessidade fisiológica (fome, sede, sono, sexo), com 11 questões; 2) necessidade de segurança (defesa, proteção, salário, casa própria, seguro saúde, aposentadoria, emprego), com 4 questões; 3) necessidade de afiliação (relacionamento, amor, pertencer a um grupo), 9 questões; 4) necessidade de autoestima (autoestima, reconhecimento, status, prestígio), 9 questões, e 5) necessidade de autorrealização (realização de potencialidades e a expressão do crescimento pessoal), nove questões.

Em cada um dos âmbitos e necessidades, os participantes da pesquisa assinalaram o número que indicava seu grau de satisfação, a partir da afirmação de cada enunciado, com opções (1) discordo muito = ÓTIMO; (2) discordo = BOM; (3) nem discordo/nem concordo = REGULAR; (4) concordo = RUIM; (5) concordo muito = PÉSSIMO. Também foram obtidas informações quanto às variáveis gênero, idade e tempo de atuação profissional na área. Para verificar o nível de satisfação em cada âmbito, foi realizada a soma das respostas pelo número de questões, sendo o escore mínimo de 11 para ótimo e máximo de 25 para péssimo, respectivamente. 


\section{Coleta de Dados}

Para a coleta de dados, foi realizado um contato prévio junto aos coordenadores das academias selecionadas, para uma breve explicação sobre o estudo, solicitando a participação dos profissionais de Educação Física, e para agendar o dia da coleta de dados. No dia da coleta de dados, foi solicitada a assinatura do Termo de Consentimento Livre e Esclarecido (TCLE) pelos participantes; em seguida, o questionário foi entregue aos participantes pelos pesquisadores, que fizeram uma breve explicação sobre o instrumento. A coleta de dados aconteceu entre junho e agosto de 2016.

\section{Análise dos Dados}

A análise dos dados foi realizada mediante uma abordagem de estatística descritiva e inferencial, com o auxílio do Software SPSS 22.0. Para as variáveis numéricas, inicialmente foi verificada a normalidade dos dados, por meio do Teste de Shapiro-Wilk. Como os dados apresentaram distribuição normal, foram utilizados Média (x) e Desvio padrão (dp) para a caracterização dos resultados. Na comparação do nível de satisfação e das necessidades de satisfação no trabalho, em função do gênero (masculino e feminino) e do tempo de atuação na ginástica de academia (menos de cinco anos e cinco anos ou mais), foi utilizado o teste $t$ de Student independente. Considerou-se o nível de significância de $p<0,05$.

\section{Resultados}

A média de idade do grupo de participantes era de $26,9 \pm 4,56$. De acordo com os resultados apresentados na Tabela 1, nota-se que, de uma forma geral, os professores de ginástica de academia apresentaram nível de satisfação regular na regência das aulas de ginástica de academia ( $x=19,08 ; d p=4,01$ ) e inferiores (ruim e péssimo), nas necessidades de autorrealização $(x=4,48 ; d p=1,10)$.

\section{Tabela 1}

Análise descritiva do nível de satisfação e das necessidades de satisfação na regência de aulas de ginástica de academia

\begin{tabular}{lccc}
\hline SATISFAÇÃO NO TRABALHO & Mínimo & Máximo & $\mathbf{x} \pm \mathbf{d p}$ \\
\hline Necessidades fisiológicas & 2,27 & 4,45 & $3,50 \pm 0,63$ \\
Necessidades de segurança & 1,25 & 5,00 & $3,55 \pm 1,08$ \\
Necessidades de afiliação & 1,00 & 3,87 & $3,88 \pm 1,20$ \\
Necessidade de autoestima & 2,22 & 5,33 & $3,87 \pm 0,84$ \\
Necessidades de autorrealização & 2,00 & 6,00 & $4,28 \pm 1,10$ \\
Nível de satisfação & 9,94 & 25,53 & $19,08+4,01$ \\
\hline
\end{tabular}

x: média; dp: desvio padrão.

Ainda com base na Tabela 1, constata-se que as necessidades fisiológicas e de segurança apresentaram um melhor escore em relação às outras necessidades.

Ao comparar os níveis em relação ao gênero (Tabela 2), encontrou-se diferença significativa nos âmbitos fisiológicos $(p=0,034)$ e de segurança $(p=0,043)$, evidenciando que os 
homens apresentaram menor satisfação no trabalho em relação aos aspectos fisiológicos e de segurança, quando comparados com as mulheres.

\section{Tabela 2}

Comparação do nível de satisfação e das necessidades de satisfação na regência de aulas de ginástica de academia em função do gênero

\begin{tabular}{lccc}
\hline \multirow{2}{*}{ SATISFAÇÃo NO TRABALHO } & Feminino $(\mathbf{n}=\mathbf{1 1})$ & Masculino $\mathbf{( n = 9 )}$ & \multirow{2}{*}{$\mathbf{P}$} \\
\cline { 2 - 3 } & $\mathbf{x} \pm \mathbf{d p}$ & $\mathbf{x} \pm \mathbf{d} \mathbf{p}$ & \\
\hline Necessidades fisiológicas & $3,24 \pm 0,54$ & $3,82 \pm 0,60$ & $0,034^{*}$ \\
Necessidades de segurança & $3,11 \pm 1,14$ & $4,08 \pm 0,76$ & $0,043^{*}$ \\
Necessidades de afiliação & $3,77 \pm 1,17$ & $4,01 \pm 1,30$ & 0,663 \\
Necessidade de autoestima & $3,55 \pm 0,77$ & $4,25 \pm 0,81$ & 0,066 \\
Necessidades de autorrealização & $3,93 \pm 0,94$ & $4,71 \pm 1,17$ & 0,114 \\
Nível de satisfação & $17,61 \pm 3,83$ & $20,88 \pm 3,66$ & 0,068 \\
\hline
\end{tabular}

* Diferença significativa: $p<0,05$ - Teste $t$ de Student independente.

x: média; dp: desvio padrão.

Em relação aos demais âmbitos, necessidades de afiliação, autoestima, autorrealização e no nível total de satisfação no trabalho, não foi encontrada diferença significativa $(p<0,05)$ em relação ao gênero. Entretanto, constatam-se índices maiores de satisfação, nestes âmbitos, nos professores do gênero feminino.

Ao comparar o nível de satisfação e das necessidades de satisfação na regência de aulas de ginástica de academia, em função do tempo de atuação (Tabela 3), não foi encontrada diferença significativa $(p<0,05)$ entre os grupos em nenhuma das necessidades e nem no nível total de satisfação.

\section{Tabela 3}

Comparação do nivel de satisfação e das necessidades de satisfação na regência de aulas de ginástica de academia em função do tempo de atuação

\begin{tabular}{|c|c|c|c|}
\hline \multirow{2}{*}{ SATISFAÇÃO NO TRABALHO } & Menos de 5 anos $(n=10)$ & 5 anos ou mais & \multirow{2}{*}{$\mathbf{P}$} \\
\hline & $x \pm d p$ & $x \pm d p$ & \\
\hline Necessidades fisiológicas & $3,53 \pm 0,64$ & $3,48 \pm 0,65$ & 0,872 \\
\hline Necessidades de segurança & $3,52 \pm 1,20$ & $3,57 \pm 1,02$ & 0,921 \\
\hline Necessidades de afiliação & $3,92 \pm 1,27$ & $3,83 \pm 1,20$ & 0,874 \\
\hline Necessidade de autoestima & $3,92 \pm 0,96$ & $3,81 \pm 0,75$ & 0,771 \\
\hline Necessidades de autorrealização & $4,40 \pm 1,04$ & $4,16 \pm 1,19$ & 0,638 \\
\hline Nível de satisfação & $19,30 \pm 4,09$ & $18,86 \pm 4,15$ & 0,814 \\
\hline
\end{tabular}

x: média; dp: desvio padrão.

\section{Discussão}

Como importantes achados deste estudo, encontrou-se que os professores de ginástica em academia apresentaram satisfação regular na regência das aulas e índices inferiores (ruim e péssimo) nas necessidades de autorrealização. 
Ao analisarem as necessidades de autorrealização, Cattani, Vilas Boas e Conceição (2003) e Garlet et al. (2007) apontam que somente podem ser satisfeitas por recompensas, que são dadas intrinsecamente pelas pessoas a si próprias (como o sentimento de realização tornando seu desejo real). Nas situações de trabalho, Santos (1996) e Moreira, Ferreira, e Ferreira (2014 ) apontam que estas podem se apresentar: 1) na forma de satisfação pelo bom ambiente; 2) na realização por metas pessoais e organizacionais alcançadas; 3) no comprometimento no trabalho; 4) no orgulho por poder participar nas decisões no trabalho, e 5) na oportunidade de crescimento e valorização do potencial da própria pessoa.

No contexto das academias de ginástica, estudos realizados por Espírito-Santo e Mourão (2006) e Coelho Filho e Votre (2010) apontam que a padronização das aulas de ginástica de academia possibilita aos donos dos estabelecimentos trocar os profissionais com facilidade e isso se reflete na satisfação e autorrealização dos profissionais, uma vez que acabam perdendo benefícios em termos salariais e sendo submetidos a situações de trabalho sem registro ou planos de carreira.

A satisfação dos professores é resultado de um conjunto de fatores inter-relacionados, fazendo que o mesmo busque conhecimentos, interação social, profissionalismo para ministrar as aulas, ser prestativo com a equipe, estar satisfeito com o salário pelo tempo de atuação e formação profissional. Entretanto, nota-se, com base em Farias Lemos, Both, Nascimento, e Folle (2008), Moreira, Nascimento, Sonoo, e Both (2010) e Martins e Figueiredo (2015), que a remuneração tem se tornado uma preocupação dos profissionais da área e acaba gerando insatisfação no trabalho, uma vez que, na maioria das vezes, esta é incompatível com seu nível de capacitação percebido, qualidade do serviço prestado e carga horária de trabalho.

Já o fato de as necessidades fisiológicas e de segurança apresentarem um melhor escore em relação às outras necessidades pode se dar em razão de que, segundo Cattani et al. (2003), tais necessidades levam a pessoa a proteger-se de qualquer perigo físico ou abstrato, qual seja: estabilidade funcional, condições seguras de trabalho (equipamento - doenças), plano de saúde, seguro de vida e possibilidade de ascensão (plano de carreira). Observe-se que a falta destas condições pode gerar ausência e demissão, exatamente por ameaçar as condições mínimas de sobrevivência.

Espírito-Santo e Mourão ( 2006), em um estudo realizado com seis profissionais de Educação Física atuantes em uma academia de ginástica na cidade do Rio de Janeiro, constataram que a instabilidade dos empregos, provocada pela falta de vínculos empregatícios e, ainda, a remuneração por hora/aula, leva os profissionais a acumularem uma grande carga de trabalho. Nota-se, com relação ao assunto, uma precariedade de condições de trabalho do profissional de Educação Física nas academias de ginástica, o que tem levado a quadros de dores musculares, altos índices de acidente de trabalho, doenças ocupacionais e doenças psíquicas (depressão, burnout) (Watson, 2003 ).

Sobre o fato de os homens apresentarem menor satisfação no trabalho em relação aos aspectos fisiológicos e de segurança, quando comparados com as mulheres, os achados da presente pesquisa contrapõem-se aos evidenciados na literatura.

Estudos realizados por Coelho Filho (2000), Espírito-Santo e Mourão (2006), e Coelho Filho e Votre (2010) apontam que as grandes academias tendem a privilegiar o profissional 
do gênero masculino, apresentando uma restrição à mulher profissional de ginástica, bem como ao profissional mais velho, o que pode vir a viabilizar melhor satisfação fisiológica e de segurança aos professores do gênero masculino, no contexto das academias de ginástica.

Em relação aos demais âmbitos - necessidades de afiliação, autoestima, autorrealização e no nível total de satisfação no trabalho -, não foi encontrada diferença significativa ( $p<$ $0,05)$ em relação ao gênero. Entretanto, percebem-se índices um pouco maiores de satisfação no gênero feminino.

O fato de não haver diferença significativa no nível de satisfação e das necessidades de satisfação na regência de aulas de ginástica de academia em função do tempo de atuação indica que essa variável, na situação analisada, não foi um elemento interveniente na satisfação no trabalho dos professores na regência de aulas de ginástica de academia.

Uma explicação para tal seria que as pessoas se tornam mais satisfeitas e realistas com a vida, com o passar dos anos, incluindo satisfação com o trabalho (Martinez \& Paraguay, 2003). E, por estar na fase de estabilização e consolidação (quatro a nove anos de trabalho) ( Both, Nascimento, \& Borgatto, 2007), o profissional acaba por estabilizar suas ações, adquirindo confiança interventiva e explorando as diferentes possibilidades de atuação, buscando consolidar seu repertório profissional, além de diversificar suas atividades, posturas, certezas e incertezas, tornando-se mais motivados, dinâmicos e mais empenhados.

Diante dos dados apresentados, constata-se que os profissionais de Educação Física que ministram aulas de ginástica de academia mostraram-se com níveis de satisfação entre regular e bom, estando este índice relacionado a aspectos psicossociais e ao sentido do conteúdo, no contexto individual e social (Martinez, 2002 ). Deste modo, a empresa deve se mostrar atenta para a satisfação de afiliação, autoestima e realização desses profissionais, oportunizando um plano de carreira, boa remuneração, direito trabalhista e autonomia para que o mesmo se motive intrinsecamente, o que pode refletir na melhora dos índices de autoestima e realização.

Nota-se que a maioria dos profissionais que atuam nas academias de ginástica tem uma rotina de trabalho com características de carga horária e esforço físico elevados, condições nem sempre adequadas e que, quando associadas às baixas remunerações e incertezas geradas, muitas vezes, pela informalidade do trabalho, acabam por interferir na percepção da qualidade de vida profissional e na satisfação no ambiente de trabalho.

Por fim, vale ressaltar que este estudo apresenta fatores limitantes, na medida em que reflete acerca da dinâmica de apenas quatro academias, escolhidas de forma intencional, e que outras variáveis, como características individuais, laborais e outras atividades profissionais realizadas, podem ter influenciado na satisfação dos professores avaliados. Talvez, em outro contexto e outras regiões, fosse possível perceber outros índices de satisfação em ministrar essas aulas de ginástica.

\section{Considerações Finais}

A satisfação no trabalho entre os professores de ginástica de academia encontra-se nos índices regular a bom, sendo necessário atentar-se para as questões relacionadas à necessidade de autorrealização. Quando comparados os níveis de satisfação entre os gêneros masculino e feminino, houve um índice de satisfação maior no gênero feminino. Contudo, quan- 
to menores forem os índices de autorrealização das necessidades, maiores serão a satisfação e a motivação desses profissionais em ministrar essas aulas de ginástica de academia, trazendo bons resultados e um bom desempenho em suas funções.

Por fim, diante do quadro de poucos estudos realizados sobre a temática, sugere-se que novas investigações sejam feitas aumentando-se o número amostral, bem como buscando traçar análises qualiquantitativas sobre a satisfação de profissionais de Educação Física na regência em aulas de ginástica de academia, de modo a identificar variáveis que podem influenciar nos resultados do questionário e subsidiar indicativos para profissionais e gestores melhorarem as condições laborais no contexto das aulas de ginástica em academia.

\section{Referências}

Both, J., Nascimento, J. V., Borgatto, A. F. (2007). Estilo de vida dos professores de Educação Física ao longo da carreira docente no Estado de Santa Catarina. Revista Brasileira de Atividade Física \& Saúde, 12(3), 54-64.

Brooks, R. (Ed.). (2007). Public services at the crossroads. Londres: Institute for Public Policy Research.

Cattani, A., Vilas Boas, A. A., \& Conceição, R. D. P. D. (2003) . Fatores determinantes para auto-realização: Uma análise da Empresa Bertolini. In: SLADE, Bolivia, 2005. Disonível em: https://pt.scribd.com/document/90660765/artigo02, acesso em novembro de 2018.

Coelho Filho, C. A. D. A. (2000). O discurso do profissional de ginástica em academia no Rio de Janeiro. Movimento, 6(12).

Coelho Filho, C. A. D. A., \& Votre, S. J. (2010). Imagens da prática profissional emacademias de ginástica na cidade do Rio de Janeiro. Revista Brasileira de Ciências do Esporte, 31(3), 95-110.

Espírito-Santo, G., \& Mourão, L. (2006). A auto-representação da saúde dos professores de educação física de academias. Revista Brasileira de Ciências do Esporte, 27(3), 39-55.

Farias, G. O., Lemos, C. A. F., Both, J., Nascimento, J. V do., \& Folle, A. (2008). Carreira docente em Educação Física: Uma abordagem sobre a qualidade de vida no trabalho de professores da rede estadual de ensino do Rio Grande do Sul. Journal of Physical Education, 19(1), $11-22$.

Feijó, O. G. (1998). Psicologia para o esporte. Rio de Janeiro: Shape.

Folle, A., Borges, L. J., Coqueiro, R. D. S., \& Nascimento, J. V. D. (2008). Nível de (in) satisfação profissional de professores de Educação Física da Educação Infantil. Motriz. Revista de Educação Física, 14(2), 124-134.

Garlet, A., Azevedo, A. S. D. S., Silveira, F. M. A., Dias, M., Garlet, A., Azevedo, A. S. S. \& Brasileiro, M. E. (2007). Qualidade de vida do enfermeiro que atua em Unidades de Terapia Intensiva privadas, por meio da Escala de Qualidade de Vida de Flanagan (Trabalho de Conclusão de Curso, Centro de Estudos de Enfermagem e Nutrição, Goiânia, GO).

Garay, L. C., Lima, I., \& Beresford, H. (2011). O treinamento personalizado: Um enfoque paradigmático da performance para o do bem-estar. Arquivos em Movimento, 4(1), 144-159.

Hesketh, J. L., \& Costa, M. T. P. M. (1980). Construção de um instrumento para medida de satisfação no trabalho. Revista de Administração de Empresas, 20(3), 59-68. 
Marqueze, E. C., \& Moreno, C. R. C. (2005). Satisfação no trabalho- uma breve revisão. Revista Brasileira de Saúde Ocupacional, 30(112), 69-79.

Martinez, M. C. (2002) As relações entre a satisfação com aspectos psicossociais no trabalho e a saúde do trabalhador.. (Dissertação de Mestrado, Faculdade de Saúde Pública, Universidade de São Paulo, SP).

Martinez, M. C., \& Paraguay, A. I. B. B. (2003). Satisfação e saúde no trabalho: Aspectos conceituais e metodológicos. Cadernos de Psicologia Social do Trabalho, 6, 59-78.

Martins, M. L. D. R., \& Figueiredo, Z. C. C. (2015). Trajetória formativa e profissional em Educação Física: Conhecimentos da formação inicial e perspectivas de carreira. Motrivivência, 27(44), 11-23.

Moreira, H. R., Nascimento, J. V., Sonoo, C. N., \& Both, J. (2010). Qualidade de vida do trabalhador docente em Educação Física do estado do Paraná, Brasil. Revista Brasileira de Cineantropometria e Desempenho Humano, 12(6), 435-442.

Moreira, W. L. G., Freitas, L. A. G., Guarido, E. A., \& Bruniera, C. A. V. (2017). Influência do profissional de Educação Física na manutenção de alunos de uma academia de ginástica da cidade de Londrina-PR. Revista Equilíbrio Corporal e Saúde, 8(1), 23-27.

Moreira, A., Ferreira, A. G., Ferreira, J. A. (2014). Escala de satisfação de professores de Educação Fisica: Procedimentos de construção e validade. Revista Portuguesa de Pedagogia, 48(1), 69-85. Rufino, V. S., Soares, L. F. S., \& Santos, D. L. (2000). Características de freqüentadores de academias de ginástica do Rio Grande do Sul. Kinesis, 22, 57-68.

Saba, F. (2001). Aderência: A prática do exercício físico em academias. São Paulo: Manole. Santos, F. T. (1996). Atitudes e crenças dos professores do ensino secundário: Satisfação, descontentamento e desgaste profissional. Lisboa: Instituto Inovação Educacional.

Sena, D. M. M. (2015). Ginástica de academia e metodologia do professor: Produção científica nacional 2005-2015 (Trabalho de Conclusão de Curso Bacharelado em Educação Física, Universidade Federal do Rio Grande do Sul, Porto Alegre, RS).

Siqueira, M. M., \& Gomide Jr., S. (2004). Vínculos do indivíduo com o trabalho e com a organização. In J. C. Zanelli, J. E. Borges-Andrade, \& A. V. B. Bastos (Orgs.), Psicologia, organizações e trabalho no Brasil (pp. 317-348). Porto Alegre: Artmed.

Watson, P. (2003). Sofrimento psíquico, lesões e doenças ocupacionais em profissionais de Educação Física que atuam em academias de ginástica. (Trabalho de Conclusão de Curso Licenciatura em Educação Física, Universidade Gama Filho, Rio de Janeiro, RJ).

Recebido: $31 / 07 / 2017$

Última revisão: 27/11/2017

Aceite final: 27/11/2017

\section{Sobre os autores:}

Ana Luiza Barbosa Anversa- Doutora em Educação Física, Universidade Estadual de Maringá (UEM). E-mail: ana.beah@gmail.com

Tieli Fernanda Ribeiro- Bacharel em Educação Física. E-mail: fernanda.tieli@gmail.com

Luciana Boligon Refundini- Mestre em Educação Física. E-mail: luciana.refundini@unifamma.edu.br Daniel Vicentini de Oliveira- Doutor em Gerontologia. E-mail: d.vicentini@hotmail.com 
